\title{
Superior Capsular Reconstruction Using 3-layered Fascia Lata Autograft Reinforced with a Nonresorbable Suture Mesh
}

\author{
Martin Polacek, M.D., Ph.D., and Cecilie P. Nyegaard, M.D.
}

\begin{abstract}
Purpose: The purpose of this study was to evaluate the short-term clinical outcomes and the complications related to arthroscopic superior capsular reconstruction (SCR) using a 3-layered fascia lata autograft (FLA) reinforced with a nonresorbable suture mesh, in the treatment of irreparable massive rotator cuff tears (MRCTs). Methods: Consecutive patients with irreparable MRCTs (Goutallier 4, Hamada grade 1-2, Bateman 3-4) who were treated with arthroscopic SCR using reinforced FLA in 2018 were included. Patients with an irreparable subscapularis/infraspinatus, serious cardiovascular condition, systemic infection, rheumatic disease, and known alcohol/drug abuse were excluded from the study. Range of motion and Shoulder Pain and Disability Index (SPADI) scores were assessed preoperatively, at 6 months, and at 12 months postoperatively. A simple $t$ test was used to compare the outcomes. $P$ values $<.05$ were considered significant. Complications and revision surgeries were registered. Results: A total of 24 consecutive patients (15 males, 9 females) with a mean age of 61 years (range, 41-76) were enrolled. Twenty (83\%) patients achieved minimal clinically important difference in the SPADI score $(>18)$ and $14(58 \%)$ experienced substantial clinical benefit in SPADI $(>45)$. The mean SPADI score improved from 59.0 to $9.7(P<.0001)$ at 1 -year follow-up. Active abduction improved from $59.5^{\circ}$ to $154.3^{\circ}$ $(P<.0001)$ and active forward flexion improved from $67.0^{\circ}$ to $160.3^{\circ}(P<.0001)$ at 1 -year follow-up. Eighteen $(75 \%)$ patients achieved substantial clinical benefit in active abduction $\left(>28.5^{\circ}\right)$ and active forward flexion $\left(>35.4^{\circ}\right)$. Complications included progression of osteoarthritis in 2 cases, tear of the graft in 1 case, and pullout of the anchor in another. Two patients experienced donor site morbidity after harvesting the fascia lata autograft. Conclusions: Eighty-three percent of the patients achieved minimal clinically important difference and were successfully treated with arthroscopic SCR using a FLA reinforced with a suture mesh. The procedure had a complication rate of $17 \%$ and $8.5 \%$ of the patients experienced donor site morbidity. All complications occurred in patients who were previously treated with an attempted rotator cuff repair. Level of Evidence: Level IV, therapeutic case series.
\end{abstract}

D espite improved surgical techniques and therapeutical approaches, the treatment of massive rotator cuff tears (MRCTs) remains challenging and many MRCTs are considered irreparable. ${ }^{1-3}$ The

From the Orthopedic Department, Drammen Hospital, Vestre Viken Hospital Trust, Drammen, Norway (M.P.); and Fjell Medical Center, Drammen, Norway (C.P.N.).

The authors report the following potential conflicts of interest or sources of funding: M.P. received personal fees and nonfinancial support from Arthrex. Full ICMJE author disclosure forms are available for this article online, as supplementary material.

Received December 18, 2019; accepted May 18, 2020

Address correspondence to Martin Polacek, M.D., Ph.D., Orthopedic Department, Drammen Hospital, Vestre Viken Hospital Trust, 3004 Drammen, Norway.E-mail:marpol@vestreviken.no

(C) 2020 THE AUTHORS. Published by Elsevier Inc. on behalf of the Arthroscopy Association of North America. This is an open access article under the CC BY-NC-ND license (http://creativecommons.org/licenses/by-nc-nd/4.0/). 2666-061X/191546

https://doi.org/10.1016/j.asmr.2020.05.011 definition of irreparability has been inconsistent and changes with the development of new surgical instruments and surgical techniques. ${ }^{4-6}$ One can assume that a tear is definitively irreparable if the complete repair of the tendon is not physically possible during surgery. ${ }^{5,7-9}$ Some claim that the irreparability can be predicted based on the preoperative magnetic resonance imaging (MRI) findings or a likelihood of poor outcome after the repair., ${ }^{4,10,11}$ Though, in such a situation, the possibility of actually achieving an intraoperative repair is not considered, and thus some of the patients might be denied the possibility to improve without using the prosthetic option. ${ }^{4,7,10,12}$

An initial treatment in irreparable MRCTs is usually conservative and includes nonsteroidal antiinflammatory drugs, subacromial corticosteroid injections, and physiotherapy. ${ }^{5}$ The most common surgical technique in the treatment of irreparable MRCTs is arthroscopic debridement. ${ }^{13}$ Earlier studies 
showed an approximately $85 \%$ success rate at shortterm follow up. ${ }^{13}$ However, in the long term, the results deteriorated. ${ }^{14}$ Other techniques include biceps release, biceps tenodesis, partial tendon repair, augmentation or bridge grafting of the rotator cuff tendon, and several tendon transfers. ${ }^{5,15-20}$

Although the role of the anterior and posterior joint capsule in the shoulder joint has been well recognized, the role of the superior capsule has been uncertain and overshadowed by the role of the superior rotator cuff. ${ }^{21-23}$ Biomechanical testing has demonstrated that a tear in the superior joint capsule might lead to an abnormal load on the rotator cuff, significantly increase the glenohumeral translation in all directions and subacromial contact pressure. ${ }^{24}$ Thus, reconstructing the superior joint capsule could restore a superior translation of the humeral head and subacromial and glenohumeral contact pressure that might be disrupted by the supraspinatus tear. ${ }^{24}$ Reconstruction of the superior joint capsule using a fascia lata autograft (FLA) in the treatment of irreparable MRCTs has been described by Mihata et al. ${ }^{25}$ In this study, the graft was attached to the superior glenoid and the humeral head by suture anchors and to the infraspinatus tendon using side-toside sutures. The procedure led to a significant improvement in shoulder function after 2 years' followup, high grade of patient satisfaction, and a low complication rate. ${ }^{25}$ In an effort to reduce the operation time and eventual donor-site morbidity after the fascia lata (FL) harvest, several different techniques using either dermal allograft or dermal xenograft have been proposed. ${ }^{26,27}$ The initial studies using these grafts showed variable results with a relatively high complication rate of up to $30 \%$ and a revision rate of up to $15 \% .^{26,27}$ The thickness of both allografts and xenografts used in the superior capsular reconstruction (SCR) is around $3 \mathrm{~mm} \cdot{ }^{26,27}$ On the other side, the FLA thickness is around 6 to $8 \mathrm{~mm} .^{25}$ According to the biomechanical studies, the ideal graft for the SCR is 6to 8 -mm thick and stiff so that the graft has approximately the same thickness as native rotator cuff and does not elongate during abduction. ${ }^{28}$ There are 2 approaches to obtain FLA with the right thickness. The FLA can be harvested superiorly where the proximal part of tensor fascia lata including intermuscular septa is collected and folded twice. ${ }^{25}$ If harvested more distally, the FL might be quite thin and needs to be folded 3 to 5 times to achieve the correct thickness of the graft. Thus, a large portion of FL needs to be harvested. ${ }^{29}$ These individual variations in the thickness of FLA and technical challenges related to preparation of the graft makes standard FLA not always reproducible and a reliable option..$^{25,29-31}$ In our quest to find an improved biological autograft with higher structural strength, stiffness, and thickness, we designed a new graft based on the FL and reinforced with a nonresorbable suture mesh. We aimed to make a stiffer graft with right thickness, but without harvesting a large portion of the FL or disrupting the superior attachment of the tensor fascia lata muscle.

The purpose of this study was to evaluate the shortterm clinical outcomes and the complications related to arthroscopic SCR using a 3-layered FLA reinforced with a nonresorbable suture mesh in the treatment of irreparable MRCTs.

The hypothesis was that SCR with reinforced FLA led to a significant clinical improvement with low complication rates in the majority of the patients with irreparable MRCTs at the 1-year follow-up.

\section{Methods}

In 2018, consecutive patients who were surgically treated with arthroscopic SCR using reinforced FLA for MRCTs were identified. The patients were referred to our hospital by other surgeons, general practitioners, or physiotherapists based on tentative diagnosis of rotator cuff tear. The treating surgeon at the hospital's outpatient clinic assessed the patients regarding the reparability or irreparability of the tear. The patients with irreparable tears were informed about the SCR procedure, its potential risks, and the postoperative rehabilitation. At the same time, the patients were invited to participate in our study. The patients participated in the study with their written informed consent, and the hospital's local ethical board approved the study. The inclusion criteria were based on both clinical and radiological findings. Patients with symptomatic, chronic MRCTs characterized by (1) severe shoulder weakness, (2) pain, and (3) impaired range of motion were included in the study. All of the patients had at least 12 months of symptoms and had tried physiotherapy with focus on strengthening of the deltoid and periscapular muscles for at least 6 months. MRI scans were used to assess the rotator cuff tear, focusing on the grade of tendon retraction, fat atrophy of the supraspinatus muscle, superior migration of the humeral head, and glenohumeral cartilage. The inclusion criteria based on the MRI findings were: (1) significant retraction of the supraspinatus tendon over $4 \mathrm{~cm}$ (Bateman classification grade 3-4); (2) significant fat atrophy of the supraspinatus muscle (Goutallier stage 4); (3) light or no rotator cuff arthropathy (Hamada stage 1 and 2) without acetabularization; and (4) intact or reparable subscapularis and infraspinatus tendons. ${ }^{26,32,33}$ The exclusion criteria included patients with: (1) serious cardiovascular condition (ASA 3-5); (2) patients with an acute systemic infection; (3) patients with systemic rheumatic disease using disease modifying antirheumatic drugs; (4) patients not motivated for the postoperative rehabilitation program; and (5) patients with known alcohol or drug abuse. Previous shoulder surgery was not an exclusion criterion. Because of costs 

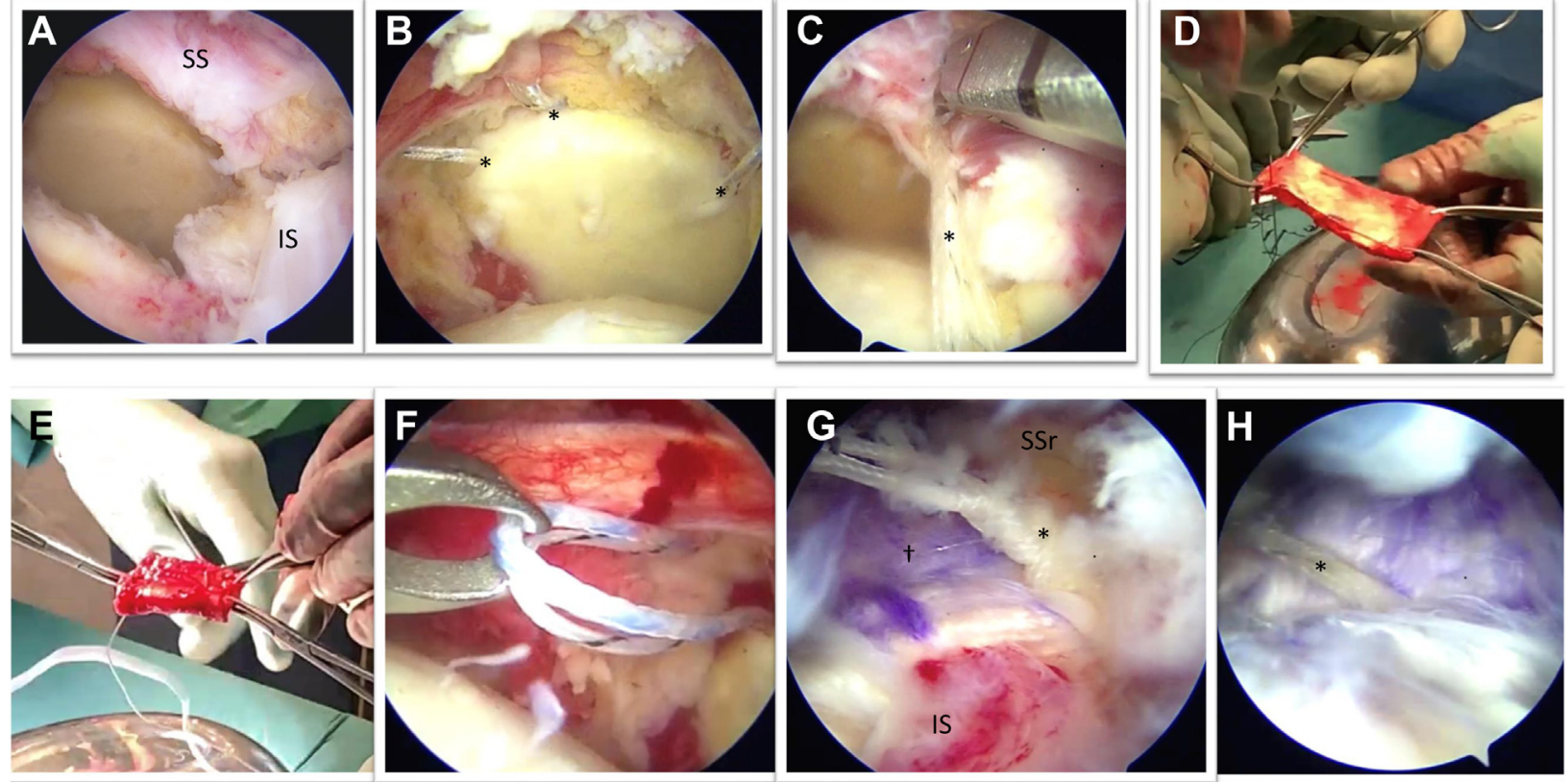

Fig 1. Superior capsular reconstruction with reinforced 3-layered fascia lata autograft. (A) Posterolateral viewing portal. The supraspinatus (SS) is retracted to the glenoid edge. The infraspinatus (IF) is not affected. A posterior interval slide is performed to improve the visualization of the superior glenoid. (B) The superior labrum is removed and the glenoid edge is prepared using a burr. Three suture anchors (Knotless suture tack, Arthrex) are placed in the superior glenoid neck $(*)$ and 2 anchors (Swivel lock 4.75 preloaded with fiber tape, Arthrex) are placed into the greater tuberosity at the cartilage margin. (C) The distance between the anchors is measured using a calibrated probe and the size of the graft is decided. The fiber tape is marked with an asterisk. (D) The fascia lata autograft (FLA) is harvested from the lateral thigh just distally to the greater trochanter and reinforced using a nonresorbable suture mesh (Mutars attachment tube, Implantcast). (E) The reinforced FLA is then folded 3 times and secured by a suture. (F) The sutures are retrieved through the lateral portal starting with lateral suture tapes in the greater tuberosity, followed by sutures from the medial glenoid anchors and passed through the graft using loose needles. The sutures from the medial glenoid anchors are then passed through the anchors' locking mechanism. The graft is shuttled into the joint and secured to the superior glenoid by tightening the sutures in the glenoid anchors. Double row configuration (Speed bridge, Swivel lock 4.75, Arthrex) is used to secure the graft to the greater tuberosity. (G) Final medial fixation of the FLA; supraspinatus remnant (SSr) is pulled over the medial superior edge of the FLA $(\dagger)$; both the FLA $(\dagger)$ and the SSr are fixed to the infraspinatus (IS) with side-to-side suture $(*)$. (H) Final lateral fixation with Fiber tapes $\left(^{*}\right)$ in double row configuration (Speed bridge, Swivel lock 4.75, Arthrex).

and availability of the hospitals MRI scanners, the postoperative imaging was not conducted in all of the patients, only in those expressing dissatisfaction with the postoperative outcomes, such as an increased pain level, shoulder weakness, and no or little functional improvement.

A single surgeon (M.P.) performed the SCR as an outpatient procedure at the hospital's day surgery unit. The size of the graft (width $\times$ length $\times$ depth/thickness) used to reconstruct the superior capsule was recorded. The treating surgeon assessed the patients preoperatively, after 6 months and at 1 year postoperatively with the Shoulder Pain and Disability Index (SPADI). A nurse at the day surgery unit administered the SPADI questionnaire preoperatively and the treating physiotherapist or the treating surgeon administered the SPADI questionnaire postoperatively. The treating surgeon also assessed the range of motion (ROM) with a goniometer (active abduction [aAbd] and active forward flexion $[\mathrm{aFF}]$ ) preoperatively and at 1 year postoperatively. The patients who had preoperative aFF and aAbd $<90^{\circ}$ were characterized as pseudoparalytic. The patients with aFF and aAbd $<90^{\circ}$ had moderate pseudoparalysis, whereas the patients with aAbd and aFF $<45^{\circ}$ had a severe pseudoparalysis. ${ }^{34-36}$ Improvement in aAbd $>28.5^{\circ}$ and aFF $>35.4^{\circ}$ at the 1 -year follow-up was considered as a substantial clinical benefit (SCB). ${ }^{37}$ Based on earlier studies, the minimal clinically important difference (MCID) in SPADI score was $18 .^{38}$ Improvement in SPADI over 45 was classified as an $\mathrm{SCB} .{ }^{37}$ The patient acceptable symptom state (PASS) was defined based on the preoperative SPADI values. $^{39}$ If the baseline SPADI was $>66$, the PASS threshold value for SPADI total was 49.2, pain 46.4, and disability 46.8. If the baseline SPADI was $<66$, the PASS threshold value for SPADI total was 39.4, pain 36.7, and disability 25.1. ${ }^{39}$ Any complications or revision surgeries were also recorded. The patients who 


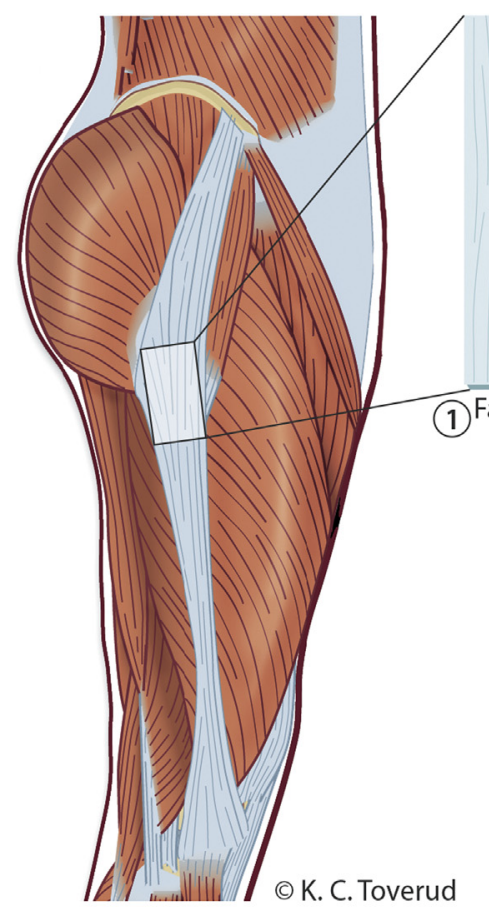

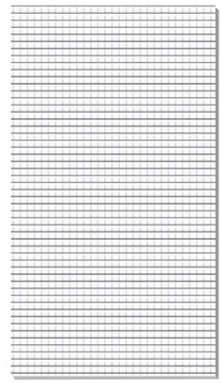

(2) Suture mesh

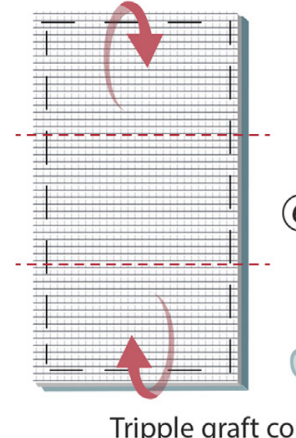

Tripple graft construction
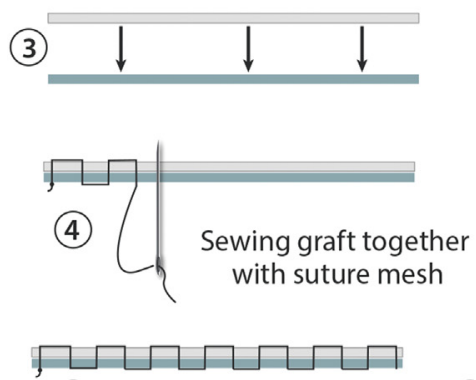

(5)

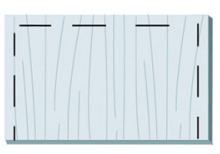

(6)

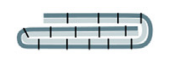

(7)

(5)

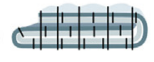

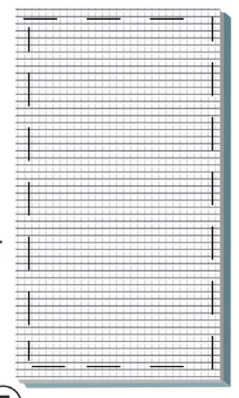

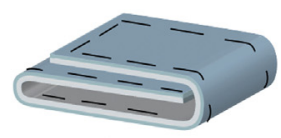

Fig 2. Preparation of the reinforced 3-layered fascia lata autograft (FLA) during arthroscopic superior capsular reconstruction. After the standard lateral incision on the upper thigh is made, the superior part of the fascia lata is visualized, the graft size is then measured, marked, and harvested (1). The FLA is reinforced with the mesh $(2,3)$ and sutured using nonresorbable sutures $(4,5)$. The graft is folded 3 times and sutured again, obtaining a graft of desired width and length $(6,7)$. (Printed with permission from (C) Kari C. Toverud.)

were in need of revision surgery were categorized as a failure in the study.

\section{Surgical Technique}

The surgical technique has been slightly modified compared to the techniques described previously (Fig 1). ${ }^{27,40}$ The procedure was performed with the patient in a lateral decubitus position with the arm in $30^{\circ} \mathrm{Abd}$ and $10^{\circ} \mathrm{FF}$. The glenohumeral joint was assessed first. The biceps tendon was released and if necessary, the subscapularis tendon was repaired. Then the subacromial space was cleaned. Acromioplasty was only performed in cases with a narrow subacromial space and in cases where the anterolateral acromion edge interfered with the placement of portals. The supraspinatus and infraspinatus tendons were tested regarding their irreparability and, if necessary, the infraspinatus tendon was repaired. The superior glenoid was prepared, removing the superior labrum and three knotless anchors (Knotless suture tack, Arthrex, Naples, FL) were inserted in the superior glenoid. Next, 2 anchors preloaded with suture tapes (Swivel lock 4.75, Arthrex) were placed in the greater tuberosity adjacent to the cartilage margin. The distance between the anchors was measured and the area of harvested fascia lata was determined. A vertical skin incision was made over the lateral part of the thigh just distally to the greater trochanter. The decided size of FL was then harvested. A nonresorbable suture mesh (Mutars attachment tube, Implantcast, Germany) was attached to the FLA and secured by a suture. The graft was then folded 3 times and secured with 1 more suture, thus preparing a reinforced 3 layered FLA of desired width, length, and thickness (Fig 2).

Sutures from each of the anchors, beginning with anchors in the greater tuberosity, were retrieved out of the lateral portal and passed through the graft using loose straight needles. The sutures from the glenoid anchors were also retrieved out of the lateral portal and passed through the graft using loose straight needles and inverted mattress stitches. The sutures were afterward passed through the locking mechanisms of the knotless self-locking glenoid anchors. The graft was shuttled into the joint and secured to the superior glenoid by tightening the sutures in the self-locking glenoid anchors. Next, the graft was secured to the greater tuberosity using double row configuration (Swivel lock 4.75, Arthrex). If possible, the supraspinatus tendon remnant was pulled over the medial superior edge of the FLA and secured to the FLA using a suture. Finally, the FLA and eventually the supraspinatus remnant was attached to the infraspinatus tendon with a side-to-side suture. The postoperative rehabilitation protocol was as follows: The shoulder was immobilized in a sling for 6 
Table 1. Functional Outcomes Following Arthroscopic Superior Capsular Reconstruction with Reinforced Fascia Lata Autograft

\begin{tabular}{|c|c|c|c|c|}
\hline & Preoperative Status & 6-Month Follow-up & 12-Month Follow-up & $\begin{array}{c}\text { Difference } \\
\text { Preoperatively vs. } 12 \text { Months } \\
\end{array}$ \\
\hline SPADI total & $59.0 \pm 19.4$ & $23.6 \pm 15.8$ & $9.7 \pm 12.3$ & $49.3 ; P<.0001$ \\
\hline SPADI pain & $63.1 \pm 19.5$ & $29.2 \pm 21.7$ & $9.3 \pm 15.0$ & $53.8 ; P<.0001$ \\
\hline SPADI disability & $57.9 \pm 20.5$ & $25.1 \pm 19.4$ & $9.9 \pm 11.2$ & $48.0 ; P<.0001$ \\
\hline
\end{tabular}

weeks. Passive ROM was initiated after 2 weeks. After 6 weeks, passive and active-assisted ROM was permitted. Exercises strengthening the deltoid and periscapular muscles were allowed after 3 months. Return to full activity was recommended at 6 to 12 months.

\section{Statistical Analysis}

The Shoulder SPADI scores, aAbd, aFF, and graft thickness were described by means and standard deviations. The dependent $t$ test was used to test for significant differences in preoperative and postoperative outcomes. $P$ values $<.05$ were considered significant.

\section{Results}

In 2018, there were 24 treated shoulders in 24 consecutive patients ( 15 males, 9 females) with a mean age of 61.25 years (range, 41-76) included in the study. One patient was excluded from the study because of an irreparable subscapularis tear. Follow-up data were obtained from all of the patients and none of the subjects were lost to follow-up. SCR was the primary procedure in 13 cases. An attempted arthroscopic rotator cuff repair was performed in 11 of the cases prior to SCR. Additional procedures during the SCR procedure included 5 cases of Infraspinatus repair, 2 cases of subscapularis repair, and 9 cases of over-the-top repair of the supraspinatus remnant. Acromioplasty was performed in 8 cases and biceps release was performed in all cases. The mean graft size in our study was width $29.8 \pm 3.0 \mathrm{~mm} \times$ length $38.0 \pm 3.2 \mathrm{~mm} \times$ depth/ thickness $7.6 \pm 1.0 \mathrm{~mm}$.

Twenty of $24(83 \%)$ patients met the criterion for MCID in SPADI score $(>18)$. Fourteen of $24(58 \%)$ patients experienced SCB (SPADI score improved $>45$ ) and 19 of $24(79 \%)$ patients achieved PASS in SPADI total, pain, and disability scores. The mean SPADI total scores improved from $59.0 \pm 19.4$ preoperatively to $23.6 \pm 15.8$ at 6 months and to $9.7 \pm 12.3(P<.0001)$ at 12 months follow-up. The mean SPADI pain score improved from $63.1 \pm 19.5$ preoperatively to 29.2 \pm 21.7 at 6 months and to $9.3 \pm 11.2(P<.0001)$ at 12 months' follow-up. The mean SPADI disability score improved from $57.9 \pm 20.5$ preoperatively to $25.1 \pm$ 19.4 at 6 months and to $9.9 \pm 11.2(P<.0001)$ at 12 months' follow-up (Table 1). Figure 3 shows a gradual improvement in functional outcomes at 6 months and 12 months postoperatively.
Active ROM improved significantly as well and 18 of $24(75 \%)$ patients experienced SCB (aAbd $>28.5^{\circ}$, aFF $>35.4^{\circ}$ ). Specifically, mean aAbd improved from $59.5^{\circ}$ $\pm 21.9^{\circ}$ to $154.3 \pm 34.6^{\circ}(P<.0001)$ and mean aFF improved from $67.0^{\circ} \pm 22.3^{\circ}$ to $160.3^{\circ} \pm 29.8^{\circ}$ $(P<.0001)$ at 1 year follow-up (Table 2$)$. All of the patients included in our study could be classified as pseudoparalytic $\left(\mathrm{aFF}<90^{\circ}\right)$ preoperatively. Eighteen patients had a moderate pseudoparalysis and 6 patients had severe pseudoparalysis $\left(\mathrm{aFF}<45^{\circ}\right)$. All of the patients with severe pseudoparalysis regained active overhead use of the arm after the SCR with an average aFF of $166.7^{\circ} \pm 16.3^{\circ}$ at final follow-up.

Postoperative complications were registered in 4 of the patients. In these cases, SCR was not the primary procedure and the patients had undergone a previous attempted rotator cuff repair. Two patients experienced superior migration of the humeral head with progression of osteoarthritis and abrasion of the graft against

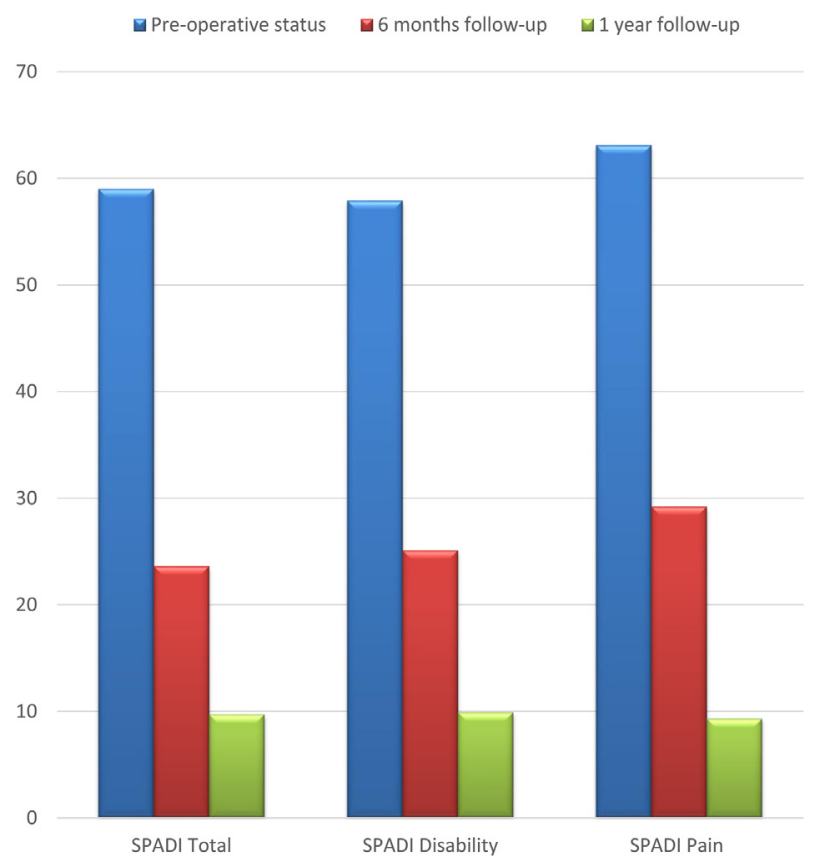

Fig 3. Shoulder function after superior capsular reconstruction using triple fascia lata autograft reinforced with nonresorbable suture mesh (Trevira, Mutars, Implantcast) assessed by the Shoulder Pain and Disability Index (SPADI). The patients were assessed with the SPADI questionnaire preoperatively, at 6 months postoperatively, and at 1 year postoperatively. 
Table 2. Range of Motion After Arthroscopic Superior Capsular Reconstruction with Reinforced Fascia Lata Autograft

\begin{tabular}{lcc}
\hline & & \\
& Preoperative Status & 12-month Follow-up \\
\hline Active abduction $\left({ }^{\circ}\right)$ & $59.5 \pm 21.9$ & $154.3 \pm 34.6$ \\
Active forward flexion $\left({ }^{\circ}\right)$ & $67.0 \pm 22.3$ & $160.3 \pm 29.8$ \\
\hline
\end{tabular}

the acromion. Revision surgery with a total reversed total shoulder arthroplasty was suggested in 1 case because of the patient's age and implantation of a subacromial balloon spacer was suggested in the second case. The third patient suffered a direct fall trauma involving her operated shoulder approximately 4 months after the surgery. The MRI scan revealed that the graft had torn from the glenoid. Because of the patient's age and persistent pain, an implantation of a reversed total shoulder arthroplasty was advised. The fourth patient had lack of functional improvement after the surgery. The MRI scan revealed a healed graft, but a pullout of the lateral, anterior anchor in the double row fixation on the humeral head. Two of these patients also experienced donor site morbidity after the FL harvest in addition to their shoulder complications. One developed a hematoma in the donor site after the surgery. The patient later presented with muscle prolapse and was treated with revision surgery where the fascia lata defect was repaired using a surgical mesh. The second patient complained of pain in the harvesting area.

\section{Discussion}

In this study, 20 of 24 patients were regarded as successfully treated. The SPADI total score improved by 49.3, SPADI pain score by 53.8 , and SPADI disability score by 48.0 . Active Abd improved by $94.8^{\circ}$ and aFF by $83.3^{\circ}$. Other surgeons have reported similar improvements in clinical outcome scores. In the initial study of 24 shoulders, Mihata et al. ${ }^{25}$ showed the same success rate of $83 \%$ ( 20 of 24 shoulders). The patients reported significant improvement in their shoulder function. Specifically, the American Shoulder and Elbow Surgeons (ASES) scores improved by 69.4 points, active elevation increased by $64^{\circ}$, and external rotation by $14^{\circ}$. A more recent study from Mihata et al. ${ }^{31}$ reported the outcomes of 100 patients treated with SCR using fascia lata autograft. The majority of patients also reported significant improvements in postoperative ASES scores (ASES $>90$ at final follow-up) and active elevation $\left(>146^{\circ}\right.$ at final follow-up). De Campos Azevedo et al. $^{30}$ reported the outcomes of 22 patients with chronic MRCTs treated with SCR using FLA. As in our study, the patients experienced significant improvement in shoulder scores and ROM. The mean Shoulder Simple Test scores improved by 6.5, Subjective Shoulder Value (SSV) improved by 37\%, and Constant Score improved by 47.4 . The mean active elevation improved by $69^{\circ}$, aAbd by $67.5^{\circ}$, active external rotation by $22.4^{\circ}$, and active internal rotation by 2.6 points.

To avoid the possibility of donor site morbidity, the techniques using dermal allograft or dermal xenograft have been established. SCR procedures using these grafts reveal a similar improvement in clinical outcome scores compared to our study. ${ }^{9,26,27}$ On the other side, improvement in ROM was less satisfactory. Denard et al. reported the outcomes of 59 patients treated with the SCR procedure using an acellular dermal allograft. ${ }^{26}$ Active FF improved by $28^{\circ}$ and external rotation improved by $19^{\circ}$. The visual analog scale improved by 4.1, the ASES score improved by 33.9 and the SSV score improved by 41.3. Pennington et al. reported outcomes in 86 patients treated with SCR surgery using a dermal allograft. ${ }^{9}$ The patients reported similar improvement in ASES shoulder scores (from 52 to 82) compared to our study; however, improvements in ROM were lower than ours (aAbd improved by $56^{\circ}$ and aFF by $39^{\circ}$ ). Polacek ${ }^{27}$ reported the outcomes of 19 patients (20 shoulders) treated with SCR using a 3-mm-thick dermal porcine xenograft. The patients experienced significant improvement in shoulder scores and ROM that were similar to our results (aAbd improved by $83.6^{\circ}$, aFF by $82.8^{\circ}$, and SPADI scores improved by 40.9 ).

Four patients in our study did not experience an improvement in shoulder function after the SCR. 1 patient teared the graft from the glenoid because of a fall trauma, 2 patients experienced a superior migration of the humeral head with progression of osteoarthritis and abrasion of the graft, and 1 patient suffered from pullout of the anchor placed in the humeral head. We registered that in all the cases with complications, SCR was performed as a secondary procedure after an attempted supraspinatus tendon suture. Of the 24 patients included in our study, 11 had undergone such a procedure before SCR. Our results indicate that previous attempted supraspinatus repair might increase the risk of failure after SCR (4 of $11 ; 37 \%$ ). None of the cases where SCR was conducted as a primary procedure was regarded as failures. Other authors using FLA reported similar complication rate compared with our results. Mihata et al. ${ }^{25,31}$ reported $16 \%$ complication rate in 2 studies including retears of the repaired infraspinatus tendons, tears of the autograft, deep infections, suture anchor pullouts, and joint contractures. De Campos Azevedo et al. ${ }^{30}$ reported a low complication rate with only 1 postoperative infection, but the MRI scans showed a graft tear in $9.1 \%$ of the cases at 6 
months' follow-up. Authors using allograft and xenograft reported higher complication rate compared to ours. Denard et al. showed $32.2 \%$ complication rate. The complications were mostly graft related and only $45 \%$ of the grafts were healed on postoperative MRI scanning. Other complications included infections and persistent pain after the biceps tenodesis. ${ }^{26}$ Polacek et al. reported a similar complication rate of $30 \%$ when using a dermal xenograft. An additional 10\% did not experience any clinically significant improvement in shoulder scores. All of the reported complications were graft related, including acute immunological rejections of the graft and graft tears/lack of healing. ${ }^{27}$ Pennington et al. ${ }^{9}$ also using dermal allografts, showed a complication rate that was much lower compared both to our results and other studies using allograft/xenograft/FLA. The author reported only a few complications and a failure rate of $4.5 \%$. Postoperative MRIs were conducted only in patients reporting dissatisfaction; thus, no healing rate of the graft has been reported. ${ }^{9}$ This could indicate that more experience with the procedure, better indications and improved surgical technique might play a role.

Defects in the superior joint capsule together with tears of the supraspinatus and infraspinatus tendon might lead to superior instability of the glenohumeral joint. ${ }^{21,41}$ The superior instability might subsequently lead to pain, weakness, and impairment of the shoulder active ROM. Severe impairment of active shoulder ROM, especially aAbd and aFF, is defined as pseudoparalysis. ${ }^{35}$ The definition of pseudoparalysis has been unclear and inconsistent in different publications. ${ }^{36}$ However, the most common definition is less than $90^{\circ}$ aFF. $^{42}$ Other authors suggested 3 stages of pseudoparalysis: (1) no pseudoparalysis where aFF is $>90^{\circ}$, (2) moderate pseudoparalysis with $\mathrm{aFF}<90^{\circ}$, and (3) severe/profound pseudoparalysis with aFF $<45^{\circ} .3^{34,35}$ Mihata et al. ${ }^{35}$ reported results of 88 patients suffering from irreparable MRCTs treated with SCR. The patients were allocated into 3 groups: (1) no pseudoparalysis (45), (2) moderate pseudoparalysis (28), and (3) severe pseudoparalysis (15). A total of $96 \%$ (27 of 28 ) of the patients with moderate pseudoparalysis and 93\% (14 of 15) of the patients with severe pseudoparalysis experienced reversal of the pseudoparalysis and improvement in aFF. ${ }^{35}$ Burkhart et al. ${ }^{34}$ reported results of 10 patients diagnosed with a profound pseudoparalysis $\left(\mathrm{aFF}<45^{\circ}\right)$. All of the patients had irreparable MRCTs and were treated with arthroscopic SCR. Nine of 10 patients regained active overhead use of the arm after the surgery with an average aFF of $159^{\circ}$ at final followup. Secondary outcomes such as ASES improved from 52 to 89 , SSV from 36 to 91 , and visual analog scale from 4.6 to 0.5 at final follow-up. MRI scans showed that 7 of 10 grafts were fully healed, 3 were partially intact, and none was missing. ${ }^{34}$ In comparison, all of our patients could be characterized as pseudoparalytic as they had preoperative aFF and aAbd $<90^{\circ}$. Six patients had severe pseudoparalysis with $\mathrm{aFF}<45^{\circ}$ and 18 had moderate pseudoparalysis with aFF $<90^{\circ}$. All of the patients with severe pseudoparalysis experienced reversal of the pseudoparalysis and regained active overhead use of the arm after the surgery, with the average aFF of $166.7^{\circ}$ at final follow-up.

Graft thickness seems to be important in achieving favorable outcomes after the SCR. The thickness of both the allografts and the xenografts commonly used is around $3 \mathrm{~mm} .^{9,26,27}$ The surgeons using FLA usually recommend 6- to 8 -mm thickness of the graft. ${ }^{25,28,31,43}$ Mihata et al. ${ }^{44}$ compared the biomechanical properties of $3 \mathrm{~mm}$ thick dermal allograft with an 8-mm FLA in a shoulder cadaver model. Biomechanical testing showed that dermal allografts elongated by $15 \%$ during the experiment, whereas the length of the FLA remained unchanged. Both allografts and autografts completely restored the subacromial contact pressure and glenohumeral joint force. Only autografts were able to completely restore the superior glenohumeral stability. Allografts restored the superior stability only partially. ${ }^{44}$ Another study focused on the biomechanical properties of FLA of different thicknesses. ${ }^{28}$ Four- and 8-mm thick grafts were used in a cadaveric biomechanical model. The results revealed that the SCR using 4-mm-thick FLA led to significantly lower subacromial contact pressure, although superior translation was not improved. In contrast, the 8-mm-thick FLA led to significant improvement of both subacromial contact pressure and superior translation. ${ }^{28}$ According to the mentioned biomechanical studies, the ideal graft for SCR is 6- to 8 -mm-thick and stiff so that the graft has approximately the same thickness as a rotator cuff and does not elongate during abduction, thus restoring the superior migration of the humeral head. In his clinical practice, Mihata and colleagues always try to make the graft stiffer by reinforcing it, using non-resorbable sutures. They usually add 5 to 10 mattress sutures in the body of the graft to achieve the sufficient stiffness. ${ }^{43}$ In our study, we chose nonresorbable suture mesh of high tensile strength to reinforce the graft. The reinforcement led to a strong and stiff FLA with the desirable thickness, without harvesting a large portion of the fascia lata. The average thickness of our reinforced FLA was $\sim 7.6 \mathrm{~mm}$ and the average area of harvested fascia lata was $9 \times 4 \mathrm{~cm}$. Because of the reinforcement with the suture mesh, we were able to obtain a relatively thick graft simply by folding fascia lata 3 times. We have observed substantial individual variations in the fascia lata thickness between the patients. Although the FL itself could be weak and thin, reinforcement with the suture mesh provided a strong and reliable graft while keeping the autografts biological potential. Two other approaches of harvesting the FLA meet the graft 
thickness criteria. Mihata prefers an open superior approach and harvests the proximal part of the FL together with intermuscular septa in order to obtain a thicker graft. The graft is then folded twice. ${ }^{25,31}$ Concerns have been that disruption of the superior tensor fascia lata muscle might lead to significant donor site dysfunction, but Mihata did not report any significant donor site morbidity. ${ }^{25,31,35}$ Angelo et al. ${ }^{29}$ established a mini-invasive approach to harvest the mid part of the FL. To obtain a graft of at least 5-mm thickness, the area of FL needed was 15 to $20 \mathrm{~cm} \times 3 \mathrm{~cm}$ and the graft had to be folded 4 to 5 times. ${ }^{29}$ In our clinic, we harvest the FL superiorly, but below the proximal attachment of the tensor fascia lata muscle. Because we use a suture mesh, we are able to obtain a 7-mm-thick graft by folding the FL 3 times, leaving a smaller harvesting area of only $9 \times 4 \mathrm{~cm}$.

Donor site morbidity can be a consequence of fascia lata harvesting. However, only 2 of our patients reported donor site morbidity in our material; pain in 1 case and a muscle prolapse in the other. In addition, both patients experienced complications after the SCR. The muscle prolapse was treated with implantation of a surgical mesh to close the defect. De Campos Azavedo et al. ${ }^{30}$ reported donor site morbidity after FL harvest in 15 patients treated with SCR using FLA. The size of the harvested FL was approximately $20 \times 3 \mathrm{~cm}$. Fifty-seven percent of the patients reported that their FL harvest site bothered them. Seventy-six percent noticed local donor site changes: deformity in $9.5 \%$, pain in $38.1 \%$, numbness in $38.1 \%$, or donor site-related claudication in $4.7 \%$. However, $76.2 \%$ of the patients stated that the result of the procedure compensated for the thigh changes, and $85.7 \%$ would undergo the same procedure again. $^{30}$

\section{Limitations}

We recognize that our study has limitations. First of all, we present a material with a relatively low number of patients, without a control group. Second, the results are only short term and we do not know the surgical outcomes in the long term. Third, we do not have postoperative MRI scans of all our patients to confirm graft healing after the SCR procedure. Fourth, our treatment population is heterogeneous. Fifth, all procedures were performed by 1 surgeon.

\section{Conclusions}

Eighty-three percent of the patients achieved MCID and were successfully treated with arthroscopic SCR using a FLA reinforced with a suture mesh. The procedure had a complication rate of $17 \%$ and $8.5 \%$ of the patients experienced donor site morbidity. All complications occurred in patients who were previously treated with an attempted rotator cuff repair.

\section{References}

1. Ainsworth R. Physiotherapy rehabilitation in patients with massive, irreparable rotator cuff tears. Musculoskel Care 2006;4:140-151.

2. Ladermann A, Denard PJ, Collin P. Massive rotator cuff tears: Definition and treatment. Int Orthop 2015;39: 2403-2414.

3. Zingg PO, Jost B, Sukthankar A, Buhler M, Pfirrmann CW, Gerber C. Clinical and structural outcomes of nonoperative management of massive rotator cuff tears. J Bone Joint Surg Am 2007;89:1928-1934.

4. Hartzler RU, Burkhart SS. Superior capsular reconstruction. Orthopedics 2017;40:271-280.

5. Ladermann A, Collin P, Athwal GS, Scheibel M, Zumstein MA, Nourissat G. Current concepts in the primary management of irreparable posterosuperior rotator cuff tears without arthritis. EFORT Open Rev 2018;3: 200-209.

6. Noyes MP, Ladermann A, Denard PJ. Functional outcome and healing of large and massive rotator cuff tears repaired with a load-sharing rip-stop construct. Arthroscopy 2017;33:1654-1658.

7. Burkhart SS. Editorial commentary: Superior capsule reconstruction with dermal allograft: Achieving the goal of joint preservation. Arthroscopy 2018;34:1774-1775.

8. Burkhart SS, Nottage WM, Ogilvie-Harris DJ, Kohn HS, Pachelli A. Partial repair of irreparable rotator cuff tears. Arthroscopy 1994;10:363-370.

9. Pennington WT, Bartz BA, Pauli JM, Walker CE, Schmidt W. Arthroscopic superior capsular reconstruction with acellular dermal allograft for the treatment of massive irreparable rotator cuff tears: Short-term clinical outcomes and the radiographic parameter of superior capsular distance. Arthroscopy 2018;34:1764-1773.

10. Sheean AJ, Hartzler RU, Denard PJ, et al. Preoperative radiographic risk factors for incomplete arthroscopic supraspinatus tendon repair in massive rotator cuff tears. Arthroscopy 2018;34:1121-1127.

11. Ek ET, Neukom L, Catanzaro S, Gerber C. Reverse total shoulder arthroplasty for massive irreparable rotator cuff tears in patients younger than 65 years old: Results after five to fifteen years. J Shoulder Elbow Surg 2013;22: 1199-1208.

12. Mulieri P, Dunning P, Klein S, Pupello D, Frankle M. Reverse shoulder arthroplasty for the treatment of irreparable rotator cuff tear without glenohumeral arthritis. J Bone Joint Surg Am 2010;92:2544-2556.

13. Levy HJ, Gardner RD, Lemak LJ. Arthroscopic subacromial decompression in the treatment of full-thickness rotator cuff tears. Arthroscopy 1991;7:8-13.

14. Zvijac JE, Levy HJ, Lemak LJ. Arthroscopic subacromial decompression in the treatment of full thickness rotator cuff tears: A 3- to 6-year follow-up. Arthroscopy 1994;10: 518-523.

15. Barber FA, Hrnack SA, Snyder SJ, Hapa O. Rotator cuff repair healing influenced by platelet-rich plasma construct augmentation. Arthroscopy 201 1;27:1029-1035.

16. Boileau P, Baque F, Valerio L, Ahrens P, Chuinard C, Trojani C. Isolated arthroscopic biceps tenotomy or tenodesis improves symptoms in patients with massive 
irreparable rotator cuff tears. J Bone Joint Surg Am 2007;89:747-757.

17. Bond JL, Dopirak RM, Higgins J, Burns J, Snyder SJ. Arthroscopic replacement of massive, irreparable rotator cuff tears using a GraftJacket allograft: Technique and preliminary results. Arthroscopy 2008;24:403-409.

18. Henseler JF, Nagels J, van der Zwaal P, Nelissen RG. Teres major tendon transfer for patients with massive irreparable posterosuperior rotator cuff tears: Short-term clinical results. Bone Joint J 2013;95-B:523-529.

19. Jones CR, Snyder SJ. Massive irreparable rotator cuff tears: A solution that bridges the gap. Sports Med Arthrosc Rev 2015;23:130-138.

20. Kanatli U, Ozer M, Ataoglu MB, et al. Arthroscopicassisted latissimus dorsi tendon transfer for massive, irreparable rotator cuff tears: Technique and short-term follow-up of patients with pseudoparalysis. Arthroscopy 2017;33:929-937.

21. Adams CR, DeMartino AM, Rego G, Denard PJ, Burkhart SS. The rotator cuff and the superior capsule: Why we need both. Arthroscopy 2016;32:2628-2637.

22. Levine WN, Flatow EL. The pathophysiology of shoulder instability. Am J Sports Med 2000;28:910-917.

23. Steinbeck J, Liljenqvist U, Jerosch J. The anatomy of the glenohumeral ligamentous complex and its contribution to anterior shoulder stability. J Shoulder Elbow Surg 1998;7:122-126.

24. Mihata T, McGarry MH, Pirolo JM, Kinoshita M, Lee TQ. Superior capsule reconstruction to restore superior stability in irreparable rotator cuff tears: A biomechanical cadaveric study. Am J Sports Med 2012;40:2248-2255.

25. Mihata T, Lee TQ, Watanabe C, et al. Clinical results of arthroscopic superior capsule reconstruction for irreparable rotator cuff tears. Arthroscopy 2013;29:459-470.

26. Denard PJ, Brady PC, Adams CR, Tokish JM, Burkhart SS. Preliminary results of arthroscopic superior capsule reconstruction with dermal allograft. Arthroscopy 2018;34:93-99.

27. Polacek M. Arthroscopic superior capsular reconstruction with acellular porcine dermal xenograft for the treatment of massive irreparable rotator cuff tears. Arthroscopy Sports Med Rehabil 2019;1:e75-e84.

28. Mihata T, McGarry MH, Kahn T, Goldberg I, Neo M, Lee TQ. Biomechanical effect of thickness and tension of fascia lata graft on glenohumeral stability for superior capsule reconstruction in irreparable supraspinatus tears. Arthroscopy 2016;32:418-426.

29. Angelo A, de Campos Azevedo CI. Minimally invasive fascia lata harvesting in ASCR does not produce significant donor site morbidity. Knee Surg Sports Traumatol Arthrosc 2019;27:245-250.

30. de Campos Azevedo CI, Angelo A, Vinga S. Arthroscopic superior capsular reconstruction with a minimally invasive harvested fascia lata autograft produces good clinical results. Orthop J Sports Med 2018;6:2325967118808242.
31. Mihata T, Lee TQ, Fukunishi K, et al. Return to sports and physical work after arthroscopic superior capsule reconstruction among patients with irreparable rotator cuff tears. Am J Sports Med 2018;46:1077-1083.

32. Liotard JP, Cochard P, Walch G. Critical analysis of the supraspinatus outlet view: Rationale for a standard scapular Y-view. J Shoulder Elbow Surg 1998;7:134-139.

33. Nove-Josserand L, Edwards TB, O'Connor DP, Walch G. The acromiohumeral and coracohumeral intervals are abnormal in rotator cuff tears with muscular fatty degeneration. Clin Orthop Relat Res 2005:90-96.

34. Burkhart SS, Hartzler RU. Superior capsular reconstruction reverses profound pseudoparalysis in patients with irreparable rotator cuff tears and minimal or no glenohumeral arthritis. Arthroscopy 2019;35:22-28.

35. Mihata T, Lee TQ, Hasegawa A, et al. Arthroscopic superior capsule reconstruction can eliminate pseudoparalysis in patients with irreparable rotator cuff tears. Am J Sports Med 2018;46:2707-2716.

36. Tokish JM, Alexander TC, Kissenberth MJ, Hawkins RJ. Pseudoparalysis: A systematic review of term definitions, treatment approaches, and outcomes of management techniques. J Shoulder Elbow Surg 2017;26:e177-e187.

37. Simovitch R, Flurin PH, Wright T, Zuckerman JD, Roche CP. Quantifying success after total shoulder arthroplasty: The minimal clinically important difference. J Shoulder Elbow Surg 2018;27:298-305.

38. Roy JS, MacDermid JC, Woodhouse LJ. Measuring shoulder function: A systematic review of four questionnaires. Arthritis Rheum 2009;61:623-632.

39. Tran G, Dube B, Kingsbury SR, Tennant A, Conaghan PG, Hensor EMA. Investigating the patient acceptable symptom state cut-offs: Longitudinal data from a community cohort using the shoulder pain and disability index. Rheumatol Int 2020;40:599-605.

40. Adams CR, Denard PJ, Brady PC, Hartzler RU, Burkhart SS. The arthroscopic superior capsular reconstruction. Am J Orthop (Belle Mead NJ) 2016;45:320-324.

41. Ishihara Y, Mihata T, Tamboli M, et al. Role of the superior shoulder capsule in passive stability of the glenohumeral joint. J Shoulder Elbow Surg 2014;23:642-648.

42. Denard PJ, Ladermann A, Brady PC, et al. Pseudoparalysis from a massive rotator cuff tear is reliably reversed with an arthroscopic rotator cuff repair in patients without preoperative glenohumeral arthritis. Am J Sports Med 2015;43:2373-2378.

43. Mihata T. Editorial commentary: Superior capsule reconstruction: Grafts for superior capsular reconstruction must be thick and stiff. Arthroscopy 2019;35:2535-2536.

44. Mihata T, Bui CNH, Akeda M, et al. A biomechanical cadaveric study comparing superior capsule reconstruction using fascia lata allograft with human dermal allograft for irreparable rotator cuff tear. J Shoulder Elbow Surg 2017;26:2158-2166. 